\title{
A compact X-ray emitting binary in likely association with 4FGL J0935.3+0901
}

\author{
Zhongxiang Wang, ${ }^{1 \star}$ Yi Xing, ${ }^{1}$ Jujia Zhang, ${ }^{2,3}$ Konstantina Boutsia, ${ }^{4}$ Gege Wang, ${ }^{1}$ \\ Jithesh V., ${ }^{5}$ Kevin B. Burdge, ${ }^{6}$ Michael W. Coughlin ${ }^{\oplus}{ }^{7}$ Dmitry A. Duev ${ }^{\oplus} 7$ \\ S. R. Kulkarni, ${ }^{6,7}$ Reed Riddle ${ }^{\oplus 6}$ and Eugene Serabyn $^{8}$ \\ ${ }^{1}$ Shanghai Astronomical Observatory, Chinese Academy of Sciences, 80 Nandan Road, Shanghai 200030, China \\ ${ }^{2}$ Yunnan Observatories, Chinese Academy of Sciences, Kunming 650216, China \\ ${ }^{3}$ Key Laboratory for the Structure and Evolution of Celestial Objects, Chinese Academy of Sciences, Kunming 650216, China \\ ${ }^{4}$ Las Campanas Observatory, Carnegie Observatories, Casilla 601, La Serena, Chile \\ ${ }^{5}$ Inter-University Centre for Astronomy and Astrophysics, Pune 411 007, India \\ ${ }^{6}$ Caltech Optical Observatories, California Institute of Technology, Pasadena, CA 91125, USA \\ ${ }^{7}$ Division of Physics, Math, and Astronomy, California Institute of Technology, Pasadena, CA 91125, USA \\ ${ }^{8}$ Jet Propulsion Laboratory, California Institute of Technology, Pasadena, CA 91109, USA
}

Accepted 2020 March 3. Received 2020 March 2; in original form 2020 January 7

\begin{abstract}
4FGL J0935.3+0901 is a $\gamma$-ray source detected by the Large Area Telescope (LAT) onboard the Fermi Gamma-Ray Space Telescope. We have conducted detailed analysis of the LAT data for this source and multiwavelength studies of the source field. Its $\gamma$-ray emission can be described with a power law $(\Gamma=2.0 \pm 0.2)$ with an exponential cut-off $\left(E_{\mathrm{c}}=2.9 \pm 1.6 \mathrm{GeV}\right)$, while the flux shows significant long-term variations. From analysis of archival Neil Gehrels Swift Observatory X-Ray Telescope data, we find only one X-ray source in the LAT's $2 \sigma$ error region. Within a 3.7 arcsec radius error circle of the X-ray source, there is only one optical object down to $r^{\prime} \sim 23$ mag. Time-resolved photometry of the optical object indicates a likely $2.5 \mathrm{~h}$ periodic modulation, while its spectrum shows double-peaked hydrogen and helium emission lines (similar to those seen in accretion discs in low-mass X-ray binaries). Combining these results, we conclude that we have discovered a compact X-ray emitting binary in likely association with 4FGL J0935.3+0901, i.e. a millisecond pulsar (MSP) binary. We discuss the implication of the optical spectral features: this binary could be a transitional MSP system at a subluminous disc state, although the other possibility, the binary in a rotationpowered state showing the optical emission lines due to intrabinary interaction processes, cannot be excluded. Further observational studies will help to determine detailed properties of this candidate MSP binary and thus clarify its current state.
\end{abstract}

Key words: stars: neutron-pulsars: general-gamma-rays: stars-X-rays: binaries.

\section{INTRODUCTION}

The unprecedented capabilities of the Large Area Telescope (LAT) onboard the Fermi Gamma-Ray Space Telescope (Fermi; Atwood et al. 2009) have greatly improved our knowledge of high-energy sources in the sky. Thus far, more than $200 \gamma$-ray pulsars have been found, ${ }^{1}$ which has established pulsars as the dominant class of $\gamma$-ray sources in our Galaxy. Nearly half of them are millisecond pulsars (MSPs). They are old neutron stars spun-up due to the recycling scenario, i.e. their progenitors are believed

\footnotetext{
^E-mail: wangzx @ shao.ac.cn

${ }^{1}$ https://confluence.slac.stanford.edu/display/GLAMCOG/Publi

c+List+of+LAT-Detected+Gamma-Ray+Pulsars
}

to be low-mass X-ray binaries, in which a neutron star gains angular momentum by accreting mass transferred from a Roche lobe filling, low-mass companion star (e.g. Alpar et al. 1982; Bhattacharya \& van den Heuvel 1991). Mainly due to Fermi observations, one new type of MSP binaries, the redbacks, has been identified (Roberts 2013). These redbacks contain a $\gtrsim 0.1 \mathrm{M}_{\odot}$ companion, differing from the long-known black widow type of MSP binaries (Fruchter, Stinebring \& Taylor 1988) in that the latter have very low-mass companions $\left(<0.1 \mathrm{M}_{\odot}\right)$. Both redbacks and black widows are systems having the pulsar strongly ablate the low-mass companion, probably mainly via the pulsar wind. Currently more than 20 redbacks or candidates (in which the compact star has not been confirmed as a pulsar) have been found (see Strader et al. 2019 and references therein). These redbacks or candidates were mostly first detected in Fermi LAT observations, 
and then were identified as compact binaries from multiwavelength observations.

Intriguingly in redbacks, a companion star may overfill its Roche lobe, providing matter to form an accretion disc around the MSP. Currently there are two field redbacks, PSR J1023+0038 (Archibald et al. 2009) and XSS J12270-4859 (Bassa et al. 2014), known to be able to have such a transition between the states of being a rotation-powered radio binary MSP (disc-free) and having an accretion disc but with X-ray luminosities $\lesssim 10^{34} \mathrm{erg} \mathrm{s}^{-1}$ (i.e. a socalled subluminous disc state; Linares 2014; Strader et al. 2019). During the latter disc state, one distinguishing feature of redbacks is their enhanced $\gamma$-ray emission (compared to that in the disc-free state; e.g. Stappers et al. 2014; Xing \& Wang 2015), since generally low-mass X-ray binaries do not have detectable $\gamma$-ray emission. In addition, a distinctive bimodal count rate distribution at $\mathrm{X}$-rays is seen in the disc state, with the switching time-scales between the two count rate levels as short as tens of seconds (e.g. Patruno et al. 2014). Based on these features, several candidate redbacks have been suggested as transitional MSP systems (e.g. 3FGL J1544.6-1125, Bogdanov \& Halpern 2015; 3FGL J0427.9-6704, Strader et al. 2016a; FL8Y J1109.8-6500, Coti Zelati et al. 2019), although no state transition has been seen in them so far.

Identification of redbacks (as well as black widows) not only provides us with knowledge of the endpoints of binary evolution, helping our understanding of evolution processes in the phase of low-mass X-ray binaries (e.g. Chen et al. 2013; Benvenuto, De Vito \& Horvath 2014), but also allows to probe the interaction of a pulsar with its companion via the pulsar wind from multiwavelength studies (e.g. Bogdanov et al. 2011; Takata et al. 2014; Romani \& Sanchez 2016). Particularly what physical properties drive the transition, i.e. forming a transitional system, are not clear. Identification of a sample of redbacks as complete as possible will certainly help. In this paper, we report our discovery of a likely 2.5 -h binary in the field of a Fermi LAT source. Its optical spectrum shows emission features similar to those seen in accretion discs in X-ray binaries. Given the overall properties (particularly the optical spectral features), this binary is likely a candidate MSP binary and could even be a transitional MSP system at its subluminous disc state.

\section{MULTIWAVELENGTH DATA AND ANALYSIS RESULTS}

\subsection{FGL J0935.3+0901 and LAT data analysis}

The source J0935.3+0901 (hereafter J0935) is an unassociated source recorded in the Fermi LAT source catalogues (e.g. Acero et al. 2015; The Fermi-LAT collaboration 2019). Its variable emission (Fig. 1), revealed by $\gamma$-ray light-curve analysis, attracted our attention. Actually it is newly listed as a variable source in the Fermi LAT Fourth Source Catalog (4FGL; The Fermi-LAT collaboration 2019). We analysed the LAT data for J0935 in detail.

LAT events in the $0.1-500 \mathrm{GeV}$ band from the updated Fermi Pass 8 data base (P8R3) from 2008 August 4 15:43:36 UTC to 2018 December 2 08:29:55 UTC were selected. A region of interest (ROI) with a size of $20^{\circ} \times 20^{\circ}$ was selected for analysis, centred at the source's 4FGL position RA $=143.8326$ and Dec. $=+9.0305$ (equinox J2000.0; with a $2 \sigma$ nominal uncertainty of 0.07 ). We included the LAT events with zenith angles less than $90^{\circ}$ to prevent the Earth's limb contamination, and excluded the events with quality flags of 'bad'.

For the standard binned likelihood analysis performed to the LAT data, the source model was based on 4FGL, containing all

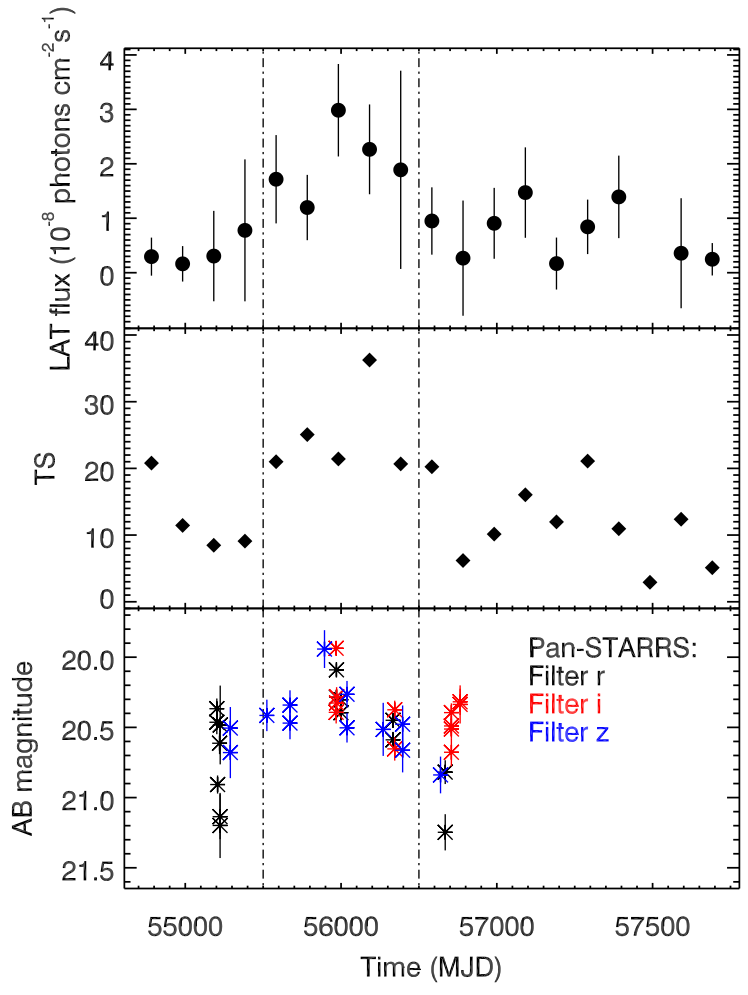

Figure 1. Top and middle panels: 200 -d binned $\gamma$-ray light curve $(0.1-$ $500 \mathrm{GeV}$ ) and test statistic (TS) curve for J0935. Flux data points with TS greater than 5 are kept. Two vertical dashed lines approximately define the high-flux time period (MJD 55500-56500). Bottom panel: the Panoramic Survey Telescope and Rapid Response System (Pan-STARRS) multiband magnitude measurements of the optical binary in the field of J0935 (see Section 2.3.1).

the 4FGL sources within the ROI. The spectral parameters of the sources within $5^{\circ}$ from J0935 were set free, and those of the remaining sources were fixed to the catalogue values. The background Galactic and extragalactic diffuse emission models included were gll_iem_v07.fits and iso_P8R3_SOURCE_V2_v1.txt, respectively. The normalizations of the diffuse components were set as free parameters.

$\gamma$-ray emission of J0935 is described with a simple power law $\mathrm{d} N / \mathrm{d} E=N_{0} E^{-\Gamma}$ in $4 \mathrm{FGL}$. In our analysis using the power law, we obtained photon index $\Gamma=2.50 \pm 0.07$ and $0.1-500 \mathrm{GeV}$ photon flux $F_{0.1-500}=12 \pm 1 \times 10^{-9}$ photons s $\mathrm{sm}^{-1}$, with a test statistic (TS) value of 233 . We also considered an exponentially cut-off power law $\mathrm{d} N / \mathrm{d} E=N_{0} E^{-\Gamma} \exp \left(-E / E_{\mathrm{c}}\right)$ for J0935, where $E_{\mathrm{c}}$ is the cut-off energy. This spectral model is characteristic of $\gamma$-ray emission of pulsars. We obtained $\Gamma=2.0 \pm 0.2, E_{\mathrm{c}}=2.9 \pm 1.6 \mathrm{GeV}$, and $F_{0.1-500}=9 \pm 2 \times 10^{-9}$ photons s${ }^{-1} \mathrm{~cm}^{-2}$, with a TS value of 237. The significance of the spectral cut-off can be estimated from $\sqrt{-2 \log \left(L_{\mathrm{pl}} / L_{\mathrm{exp}}\right)}$, where $L_{\mathrm{exp}}$ and $L_{\mathrm{pl}}$ are the maximum likelihood values obtained from a power law with and without the cut-off, respectively (Abdo et al. 2013). For J0935, we found that the spectral cut-off is $\sim 3 \sigma$ significant.

A 200-d binned light curve of J0935 in $0.1-500 \mathrm{GeV}$ was obtained by performing the maximum likelihood analysis to the data during each 200-d time intervals. A point source with exponentially cutoff power-law emission was considered. In Fig. 1, the light curve is shown. Only data points with TS greater than 5 ( $>2 \sigma$ significance) were kept in the light curve. The source's fluxes appear to have a 


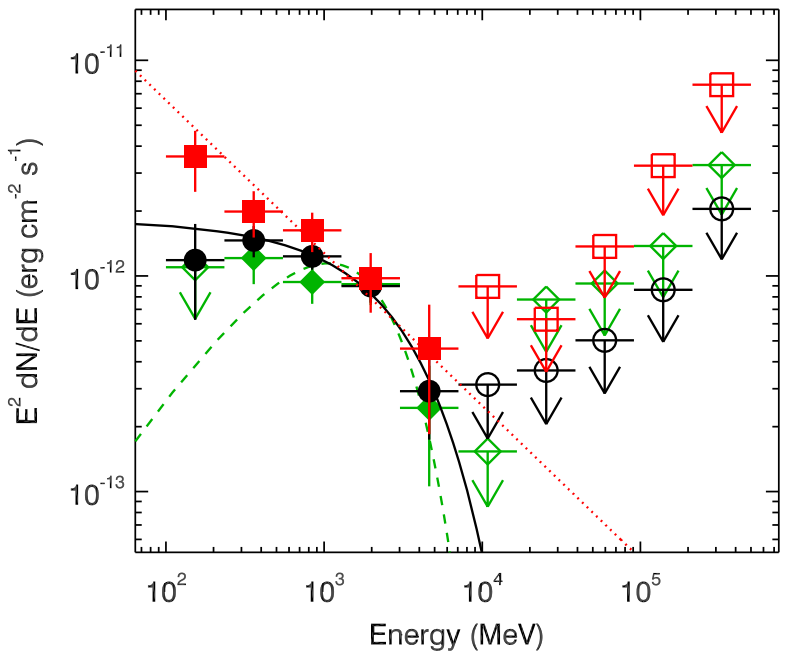

Figure 2. $\gamma$-ray spectra of J0935 in the total time period (black circles), the high-flux time period (red squares), and the low-flux time period (green diamonds), with their model fits obtained from the likelihood analysis plotted as black solid, red dotted, and green dashed curves, respectively. Open symbols are flux upper limits.

factor of 3 variation, although with large uncertainties. To verify the flux variations, we chose MJD 55500-56500 as a high-flux time period and the other time ranges as a low-flux time period, and performed likelihood analysis to the data in the two time periods, respectively. We found a power law with no cut-off better describing the emission in the high-flux time period, $\Gamma=2.7 \pm 0.1$ and $F_{0.1-500}=24 \pm 4 \times 10^{-9}$ photons s $\mathrm{cm}^{-2}$ (with a TS value of 121). In the low-flux time period, the spectral cut-off was significant $(\sim 5 \sigma), \Gamma=1.0 \pm 0.5, E_{\mathrm{c}}=1.1 \pm 0.4 \mathrm{GeV}$, and $F_{0.1-500}=$ $3 \pm 1 \times 10^{-9}$ photons s $\mathrm{s}^{-1} \mathrm{~cm}^{-2}$ (with a TS value of 107 ). Therefore, J0935 not only showed approximately a factor of 8 flux variation, but also had related spectral changes.

We extracted the $\gamma$-ray spectra of J0935 in the total time period and in the high-flux and low-flux time periods. Maximum likelihood analysis was performed to the LAT data in 10 evenly divided energy bands in logarithm from 0.1 to $500 \mathrm{GeV}$. The spectral normalizations of the sources within $5^{\circ}$ from J0935 were set as free parameters, and all the other parameters of the sources in the source model were fixed at the values obtained from the above maximum likelihood analysis. The obtained spectra are shown in Fig. 2, and the fluxes and TS values are given in Table 1, in which we kept only spectral data points with TS values greater than 5 ( $>2 \sigma$ significance), and provided 95 per cent $(2 \sigma)$ flux upper limits otherwise.

\subsection{Analysis of Swift X-Ray Telescope data}

Since positional uncertainties from Fermi LAT observations are relatively large (for which a $2 \sigma$ uncertainty region is mostly secure enough to be searched for a counterpart), we searched archival X-ray data that cover the field of J0935 for possible counterpart identification. The Neil Gehrels Swift Observatory (Gehrels et al. 2004) performed 11 short (0.1-1.0 ks) observations of the source field between 2015 January 29 and 2016 June 26. We analysed all the data taken with the X-Ray Telescope (XRT; Burrows et al. 2005) and the total exposure is $3.64 \mathrm{ks}$. The XRT data were reduced and reprocessed by applying the standard filtering and screening criteria. The XRTPIPELINE tool version 0.13 .4 in the HEASOFT package version 6.24 was used. We collected the photon counting (PC) mode data and used a sliding-cell detection algorithm in XIMAGE to detect sources. No sources were detected in any individual observations at a $3 \sigma$ significance level. We added all the observations and were able to detect two sources in the combined image (top panel of Fig. 3). Only one of them (object X) was in the Fermi LAT $2 \sigma$ error region. Using the XRTCENTROID tool, we derived the source's position, RA $=9^{\mathrm{h}} 35^{\mathrm{m}} 20^{\mathrm{s}} .60$, Dec. $=+9^{\circ} 00^{\prime} 35^{\prime \prime} .4$ (equinox J2000.0), with a positional uncertainty of 5.5 arcsec (at a 90 per cent confidence level). The position was further improved using the online tool provided by the UK Swift Science Data Centre. ${ }^{2}$ The resulting enhanced position (Goad et al. 2007; Evans et al. 2009) is RA $=9^{\mathrm{h}} 35^{\mathrm{m}} 20^{\mathrm{s}} .70$, Dec. $=+9^{\circ} 00^{\prime} 36^{\prime \prime} 8$ (equinox J2000.0), with a smaller uncertainty of 3.7 arcsec (at a 90 per cent confidence level).

Source and background events were extracted from a circular region with a radius of 24 arcsec, and the spectra of the source and background were generated from the cleaned event files with the standard grade filtering of $0-12$. The ancillary response files were also generated, using the tool XRTMKARF and the spectral redistribution matrices available in the calibration database (CALDB version 20180710). The extracted source spectrum has limited net counts (14 counts in total). We used the Cash statistics (Cash 1979) for the model fitting. An absorbed power-law model in the 0.3-10 keV energy band was used, with the hydrogen column density fixed at the Galactic value $3.6 \times 10^{20} \mathrm{~cm}^{-2}$ (HI4PI Collaboration et al. 2016). The model fit did not yield well-constrained results: power-law index is $2.3_{-1.4}^{+1.5}$ and the unabsorbed flux is $\left(1.2_{-0.7}^{+1.6}\right) \times 10^{-13} \mathrm{erg} \mathrm{cm}^{-2} \mathrm{~s}^{-1}$ (CSTAT $=8.1$ with 11 degrees of freedom).

We also searched for fast count rate variations by generating Xray light curves of object $\mathrm{X}$ with different short time bins, although the source was faint and the Swift observations are very short. No clear variations were seen. In addition, we checked the 70- and 105-month Swift Burst Alert Telescope catalogues (Baumgartner et al. 2013; Oh et al. 2018), and the source was not detected. The flux upper limit on the hard X-ray emission from the source in the 14-195 keV band thus was $\sim 10^{-11} \mathrm{erg} \mathrm{s}^{-1} \mathrm{~cm}^{-2}$.

\subsection{Optical observations and data analysis}

\subsubsection{Imaging}

The Panoramic Survey Telescope and Rapid Response System (PanSTARRS; Chambers et al. 2016; Flewelling et al. 2016) observed the field of J0935 multiple times. Only one optical object (object C; bottom panel of Fig. 3), which was variable and had magnitudes in a range of 20-21.5 (top panel of Fig. 1), was found in the Swift X-ray error circle. We note that from the Pan-STARRS measurements, object $\mathrm{C}$ showed a brightening possibly correlated with the highflux time period of J0935. In addition, the Sloan Digital Sky Survey (SDSS) observed the source field once in 2003 January. Object $\mathrm{C}$ was still the only source detected in the X-ray error circle and its multiband magnitudes are in line with the Pan-STARRS measurements (e.g. its $r^{\prime}=20.433 \pm 0.044$ in SDSS; Abazajian et al. 2009). The SDSS imaging has an upper limit of $r^{\prime} \sim 23 \mathrm{mag}$ $(3 \sigma)$ on the source field, which implies that there were no other objects in the X-ray error circle down to $r^{\prime} \sim 23 \mathrm{mag}$.

We also checked the archival data from the Palomar Transient Factory (PTF; Rau et al. 2009). The source field was well observed on 2014 February 22-23 in $R$ band, with an exposure time of $60 \mathrm{~s}$

\footnotetext{
${ }^{2}$ https://www.swift.ac.uk/user_objects/
} 
Table 1. Fermi LAT flux measurements of 4FGL J0935.3+0901.

\begin{tabular}{|c|c|c|c|c|c|c|c|}
\hline $\begin{array}{l}E \\
(\mathrm{GeV})\end{array}$ & $\begin{array}{l}\text { Band } \\
(\mathrm{GeV})\end{array}$ & $\begin{array}{l}\text { Total period } \\
F / 10^{-12} \\
\left(\mathrm{erg} \mathrm{cm}^{-2} \mathrm{~s}^{-1}\right)\end{array}$ & $\mathrm{TS}$ & $\begin{array}{l}\text { High-flux period } \\
F / 10^{-12} \\
\left(\mathrm{erg} \mathrm{cm}^{-2} \mathrm{~s}^{-1}\right)\end{array}$ & $\mathrm{TS}$ & $\begin{array}{l}\text { Low-flux period } \\
F / 10^{-12} \\
\left(\mathrm{erg} \mathrm{cm}^{-2} \mathrm{~s}^{-1}\right)\end{array}$ & TS \\
\hline 0.15 & $0.1-0.2$ & $1.2 \pm 0.6$ & 10 & $4 \pm 1$ & 20 & $<1.1$ & 0 \\
\hline 0.36 & $0.2-0.5$ & $1.5 \pm 0.2$ & 48 & $2.0 \pm 0.5$ & 21 & $1.2 \pm 0.3$ & 21 \\
\hline 0.84 & $0.5-1.3$ & $1.2 \pm 0.2$ & 84 & $1.6 \pm 0.3$ & 37 & $0.9 \pm 0.2$ & 32 \\
\hline 1.97 & $1.3-3.0$ & $0.9 \pm 0.1$ & 73 & $1.0 \pm 0.3$ & 19 & $0.9 \pm 0.2$ & 49 \\
\hline 4.62 & $3.0-7.1$ & $0.3 \pm 0.1$ & 12 & $0.5 \pm 0.2$ & 6 & $0.2 \pm 0.1$ & 6 \\
\hline 10.83 & $7.1-16.6$ & $<0.3$ & 2 & $<0.9$ & 1 & $<0.2$ & 0 \\
\hline 25.37 & $16.6-38.8$ & $<0.4$ & 0 & $<0.6$ & 0 & $<0.8$ & 0 \\
\hline 59.46 & $38.8-91.0$ & $<0.5$ & 0 & $<1.4$ & 0 & $<0.9$ & 0 \\
\hline 139.36 & $91.0-213.3$ & $<0.9$ & 0 & $<3.2$ & 0 & $<1.4$ & 0 \\
\hline 326.60 & $213.3-500.0$ & $<2.0$ & 0 & $<7.7$ & 0 & $<3.3$ & 0 \\
\hline
\end{tabular}

Note. $F$ is the energy flux $\left(E^{2} \mathrm{~d} N / \mathrm{d} E\right)$. Upper limits are quoted at a confidence level of 95 per cent.

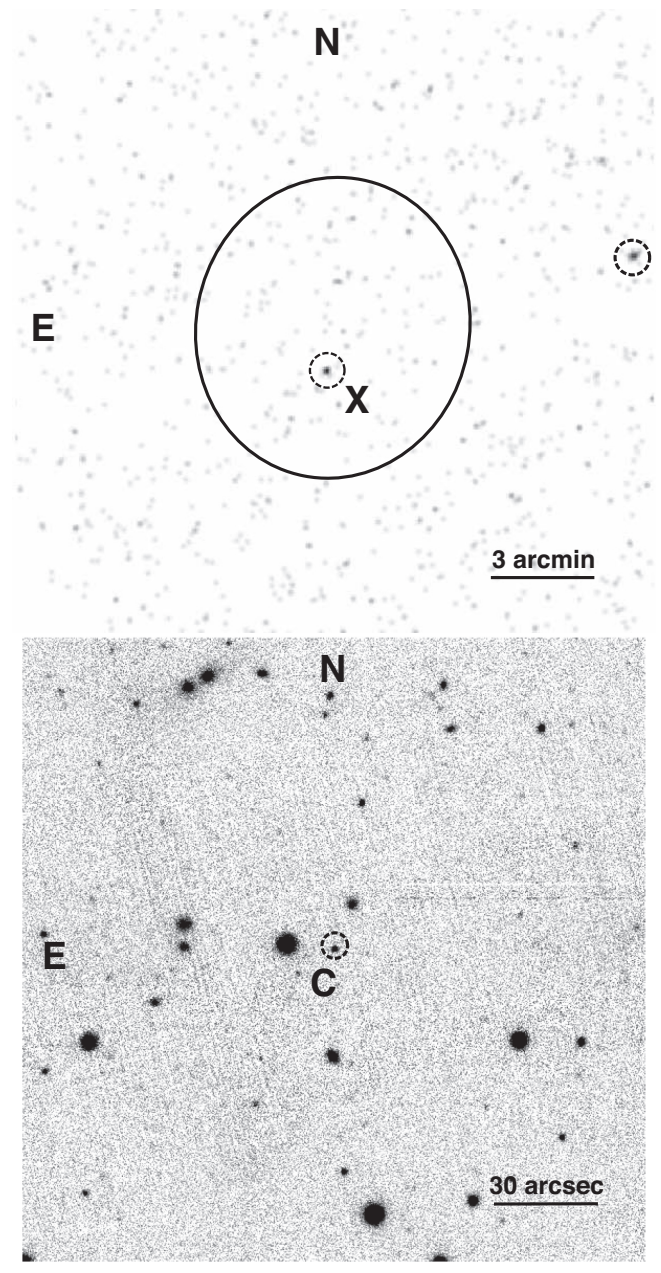

Figure 3. X-ray and optical $r^{\prime}$-band images (top and bottom panels, respectively) of the J0935 field. Two X-ray sources were detected in the field (marked by dashed circles), and one of them (object X) is in the $2 \sigma$ LAT error region (large solid ellipse). In the X-ray error circle (3.7 arcsec radius) of object $\mathrm{X}$, there is only one optical source (object $\mathrm{C}$ ) detected by the Panoramic Survey Telescope and Rapid Response System (Pan-STARRS) down to $r^{\prime} \sim 21$ mag. per frame. For the image data, we combined every three consecutive frames to increase the detection significance of object C. In total, 35 images with good point spread functions (PSFs) of sources over the two nights were obtained. We performed standard PSF fitting photometry to the object and obtained its light curve, in which nine relatively bright stars in the field without obvious variations were used for relative flux calibrations among the images. The same photometry was performed on a check star, which was chosen given its brightness similar to object $\mathrm{C}$. The light curves of object $\mathrm{C}$ and the check star are shown in Fig. 4. Comparing to the check star, significant modulation is seen in object $\mathrm{C}$. Based on the times of the modulation dips, a likely period of $\sim 2.5 \mathrm{~h}$ was found.

To confirm the periodic modulation of object $\mathrm{C}$, we further obtained a $r^{\prime}$ light curve of it with the Kitt Peak Electron Multiplying CCD (KPED) demonstrator (Coughlin et al. 2019) on 2019 April 8 . The total observation time was $3 \mathrm{~h}$. The exposure time per frame was $30 \mathrm{~s}$. KPED aperture photometry pipeline was used to measure the brightness of the object. The final light curve, shown in Fig. 4, was rebinned from the original light curve by averaging every six measurements. A sinusoidal-like modulation is seen in the light curve. We fit the light curve with a sinusoidal function, and found a period of $2.47 \pm 0.04 \mathrm{~h}$ and a semi-amplitude of $0.30 \pm 0.01 \mathrm{mag}$ ( $\chi^{2}$ is 137 with 52 degrees of freedom; see Fig. 4). Several data points near the bottom of the modulation appear to deviate away from the sinusoidal fit, which should be verified from more sensitive photometry in order to check if it is a true feature.

Sinusoidal-like modulation is commonly seen in low-mass X-ray binaries or pulsar binaries (see e.g. van Paradijs \& McClintock 1995; Strader et al. 2019). The inner face of the companion star is heated by X-ray emission or pulsar wind from the central compact object. The visible area of the heated face varies as a function of orbital phase, giving rise to the sinusoidal modulation in these binaries. The observed optical modulation thus likely indicates that object $\mathrm{C}$ is a $2.5 \mathrm{~h}$ binary. However to be cautious, because our KPED observation only lasted $3 \mathrm{~h}$, we cannot totally exclude the possibility of the light curve being ellipsoidal. Such modulation has two maxima and two minima over one orbit and is caused by the orbital motion of the companion star when there is no strong irradiation and the star is tidally distorted (e.g. van Paradijs \& McClintock 1995; also see Yap et al. 2019 for an interesting redback case changing from ellipsoidal to sinusoidal-like modulation). If this is the case, the orbital period would be $\sim 5 \mathrm{~h}$ 

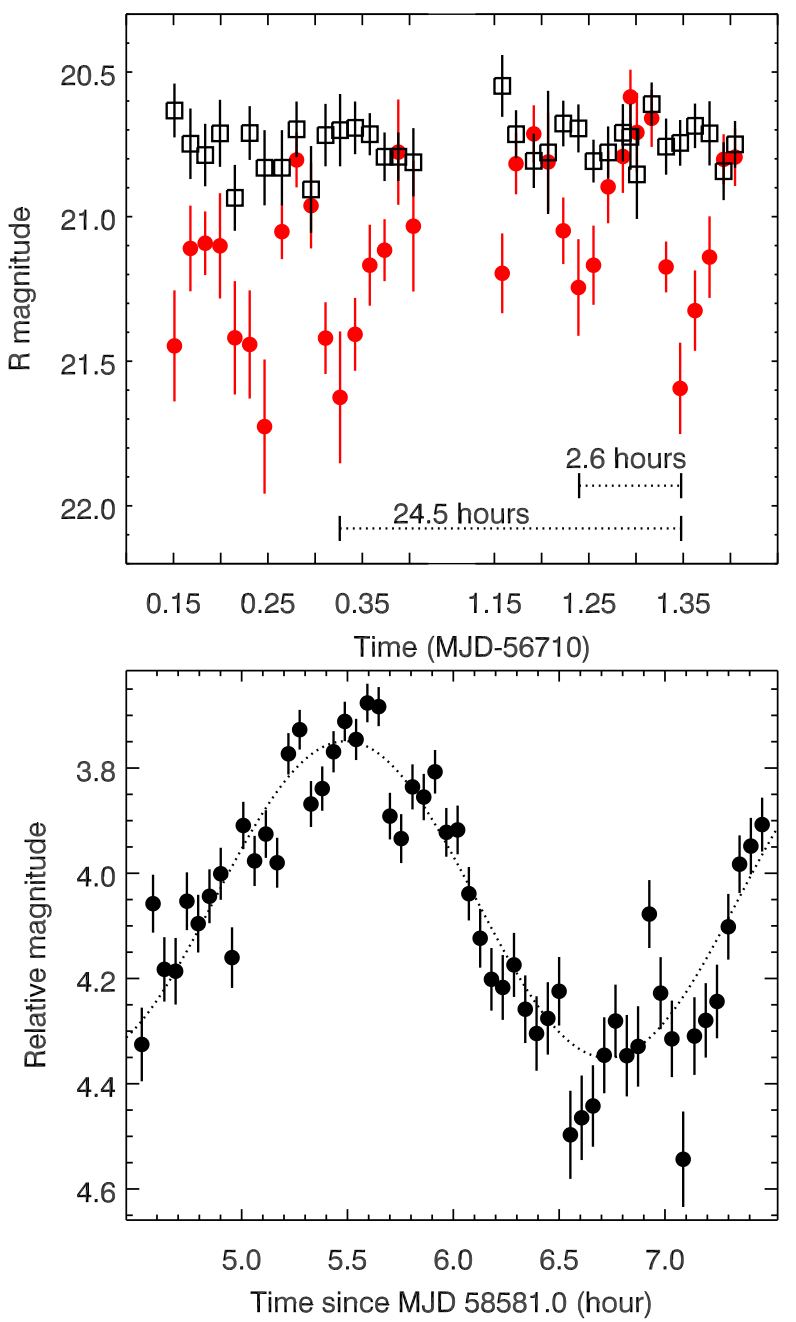

Figure 4. Top panel: the Palomar Transient Factory (PTF) $R$-band light curve of object $\mathrm{C}$ (red circles). A possible $2.5 \mathrm{~h}$ periodic modulation is seen, based on the modulation dips. For comparison, the light curve of a check star (black squares) is also shown. Bottom panel: the Kitt Peak Electron Multiplying CCD (KPED) $r$ ' light curve of object C, showing sinusoidallike modulation in detail. A sinusoidal function with a period of $2.47 \pm 0.04 \mathrm{~h}$ and a semi-amplitude of $0.30 \pm 0.01 \mathrm{mag}$ (dotted curve) can describe the modulation.

\subsubsection{Magellan spectroscopy}

On 2019 February 21, we obtained an optical spectrum of object C. The observation was conducted with the Low Dispersion Survey Spectrograph 3 (LDSS-3) on 6.5-m Magellan II (Clay) Telescope. We chose the VPH-ALL grism with a long slit of 0.75 arcsec in width, covering 4250-10000 , and providing a dispersion of $1.89 \AA$ pixel $^{-1}$.

Three 15-min exposures of the source were taken. LTT 4364, a spectrophotometric standard star, was observed for flux calibration. The three reduced spectral images were combined to yield a 45min total exposure spectrum image. The source spectrum was extracted using IRAF spectral reduction and analysis tasks. The spectrum (Fig. 5) shows prominent double-peaked $\mathrm{H} \alpha, \mathrm{H} \beta$, and three He I emission lines. These emission features are typically seen in spectra of accretion discs in X-ray binaries and also similar to that of, for example, PSR J1023+0038 in the disc state (see Wang et al. 2009). Using two Gaussian functions to fit the profiles of $\mathrm{H} \alpha$

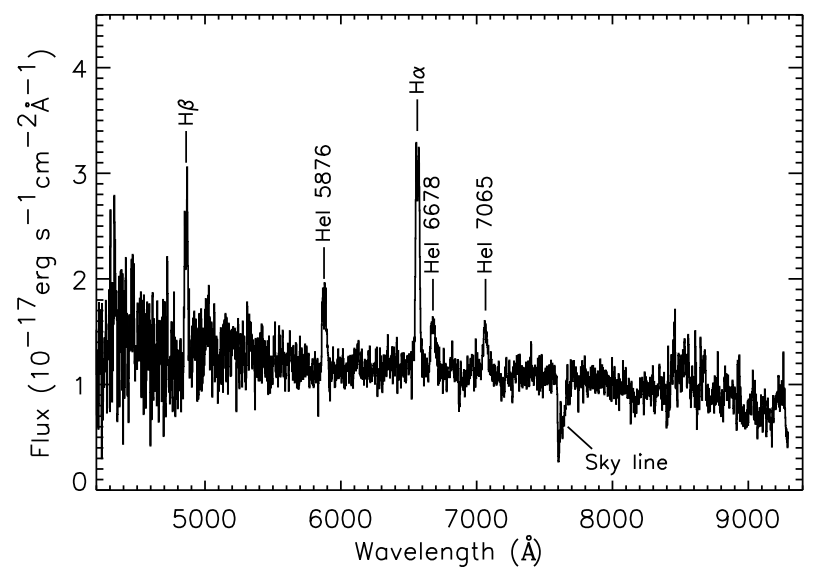

Figure 5. Magellan Low Dispersion Survey Spectrograph 3 (LDSS-3) spectrum of object $\mathrm{C}$. The detected $\mathrm{H} \alpha, \mathrm{H} \beta$, and three He I emission lines are marked.

and He I $\lambda 5876$ lines, which have clear double-peak structures, we estimated their properties. For $\mathrm{H} \alpha$, the blue and red peaks have velocities of $-430 \pm 82$ and $402 \pm 96 \mathrm{~km} \mathrm{~s}^{-1}$ [with full widths at half-maximum (FWHMs) of $463 \pm 90$ and $575 \pm 150 \mathrm{~km} \mathrm{~s}^{-1}$ ], respectively, and for $\mathrm{He} \mathrm{I} \lambda 5876$, the values are $-587 \pm 174$ and $403 \pm 276 \mathrm{~km} \mathrm{~s}^{-1}$ (FWHMs are approximately 378 and $760 \mathrm{~km} \mathrm{~s}^{-1}$, with $\sim 50$ per cent uncertainties). For comparison, these values are similar to those of PSR J1023+0038 in the disc state obtained in Wang et al. (2009).

Since the optical counterpart is faint (to the Magellan Telescope), the blue end of the spectrum suffers large uncertainties, but we suspect that there are other hydrogen and helium emission features. Also no obvious, identifiable absorption features are seen in the spectrum. These should be investigated through spectroscopy on a larger telescope. In order to confirm the likely orbital period of $2.5 \mathrm{~h}$, phase-resolved spectroscopy of absorption lines of the companion star (to obtain its radial velocity curve) is needed.

\section{DISCUSSION}

Having analysed archival X-ray and optical data and optical data obtained in our follow-up observations, we have found a binary with likely 2.5 -h orbital period in the error region of the Fermi LAT source J0935. The optical spectrum of the binary contains doublepeaked hydrogen and helium emission lines, which are typically seen in accretion discs in X-ray binaries. Given the small error circle of the X-ray source (with a radius of $3.7 \mathrm{arcsec}$ at a 90 per cent confidence level) and the binary is the only optical object in the error circle down to $r^{\prime} \sim 23 \mathrm{mag}$, it can be concluded that we have discovered a compact X-ray emitting binary. While normal compact $\mathrm{X}$-ray binaries, which for example contain an accreting neutron star, are not $\gamma$-ray sources, redbacks have both observable X-ray and $\gamma$-ray emission and particularly the transitional MSP systems in the subluminous disc state have enhanced $\gamma$-ray emission. Thus whether the 2.5-h binary is associated with J0935 determines the nature of this binary.

At high Galactic latitudes ( $b \sim 40^{\circ}$ for J0935), the dominant $\gamma$-ray sources are blazars, and their spectra are of power law, logparabola, or broken power-law shapes (e.g. Ackermann et al. 2015). For J0935, we have shown that it has a curved $\gamma$-ray spectrum, a $\Gamma \simeq 2.0$ power law with exponential cut-off $E_{\mathrm{c}} \sim 2.9 \mathrm{GeV}$. Such a spectral form is characteristic of pulsars' $\gamma$-ray emission (e.g. Abdo 
et al. 2013; Xing \& Wang 2016). The $\gamma$-ray spectrum is indeed similar to those of PSR J1023+0038 and XSS J12270-4859 in the disc state (Xing \& Wang 2015; Xing, Wang \& Takata 2018), having $\Gamma \sim 2$ and several GeV cut-off. The currently known (candidate) redbacks were mostly discovered from searches for binaries in the fields of candidate MSP systems, identified from Fermi LAT data analysis (Strader et al. 2019 and references therein). Also their $\gamma$-ray luminosities are generally in a range of $\sim 10^{33}-5 \times 10^{34} \mathrm{erg} \mathrm{s}^{-1}$, with $\mathrm{X}$-ray counterparts having luminosities of $\sim 10^{31}-10^{34} \mathrm{erg} \mathrm{s}^{-1}$. It can be noted that the (candidate) transitional systems at the disc state are mostly at the high end of the ranges. While the LAT positional uncertainties are relatively large, we found only one X-ray source, the 2.5-h binary, in the $\simeq 0.07$ ( $2 \sigma)$ error region of J0935 down to $\sim 10^{-13} \mathrm{erg} \mathrm{cm}^{-2} \mathrm{~s}^{-1}$. Therefore on the basis of the $\gamma$-ray spectral properties of J0935 and the X-ray detection in the LAT error region expected for a redback, we suggest that J0935 is associated with the 2.5-h binary. In addition, we note that a possible correlated optical brightening of the binary was seen, when the long-term $\gamma$-ray light curve of J0935 was in a high-flux time period (Fig. 1).

One notable feature of J0935 is its $\gamma$-ray flux variations, which was determined by comparing the fluxes in the two time periods we defined. This feature has not been determined to be common for redbacks or the two transitional systems. In the latter two systems, significant flux changes occurred over their state transition by appearing 'step like' (e.g. Stappers et al. 2014; Xing \& Wang 2015; Torres et al. 2017), while the same spectral form was kept. In Torres et al. (2017), $\gamma$-ray flux variations of a few redbacks (including the two transitional systems) and black widows were searched. XSS J12270-4859 in the disc state was found to have significant variations and two black widows (PSR J1446-4701 and PSR J2234+0944) also had variations. We checked and found that the $\gamma$-ray light-curve analysis methods used in Torres et al. (2017) for their search would not show J0935 as a variable (due to large flux uncertainties). In any case, the $\gamma$-ray variability of J0935 helps reinforce its compact binary nature, as isolated pulsars generally have non-variable $\gamma$-ray emission (Abdo et al. 2013).

The optical spectral features of the binary, the double-peaked emission lines, would further suggest that this is another candidate transitional MSP system at its disc state. This system would have been in the disc state all the time, since our optical spectrum was taken recently and J0935 has been in its low-flux period since 2013 August based on the long-term $\gamma$-ray light curve. However it has been noted that several redbacks (and a black widow; PSR J1740-5340A, Sabbi et al. 2003; 3FGL J0838.8-2829, Halpern, Strader \& Li 2017; 2FGL J0846.0+2820, Swihart et al. 2018; PSR J1048+2339, Strader et al. 2019; PSR J1311-3430, Romani, Filippenko \& Cenko 2015) can have strong variable emission lines and the lines (notable the $\mathrm{H} \alpha$ line) can appear to be double peaked. This feature is interpreted as the indication of material driven from the heated side of a companion by the pulsar wind and possibly plus an intrabinary shock between the companion and the pulsar wind (Strader et al. 2019 and references therein). In the subluminous disc state, the $\gamma$-ray-to-X-ray flux ratios of the transitional systems and candidates are $\sim 1$ (Coti Zelati et al. 2019; Strader et al. 2019). For J0935, its average $\gamma$-ray flux (in $0.1-500 \mathrm{GeV}$ ) is $\simeq 4.8 \times 10^{-12} \mathrm{erg} \mathrm{s}^{-1} \mathrm{~cm}^{-2}$ and the flux ratio is $\sim 40$. Although the X-ray flux is not well determined with high uncertainty, the face value of the flux ratio is more in line with those of redbacks in a rotation-powered state (Strader et al. 2019). Therefore we cannot exclude the possibility that J0935 is a rotationpowered binary MSP and whether or not it is a candidate transitional system remains to be further investigated. Optical spectroscopy with a large telescope to check the stableness of the double-peaked emission lines will help to clarify this uncertainty.

In order to confirm J0935 as a MSP binary and determine its possible transitional nature, multiband phase-resolved photometry is warranted, which allows to verify the likely 2.5 -h orbital modulation and moreover to derive detailed properties of the binary (see Strader et al. 2019 and references therein). Time-resolved photometry of PSR J1023+0038 in the disc state has revealed optical variations more complex than simple sinusoidal modulation, likely reflecting significant activity of the accretion disc (Shahbaz et al. 2015, 2018; Kennedy et al. 2018; Papitto et al. 2018). The possible deviation of the KPED light curve (at the bottom of the modulation; Fig. 4) from the sinusoidal fit might suggest similar variations in J0935. The photometry, while requiring a high cadence, will help to establish the similarity. At X-rays, a sensitive observation should be conducted, to obtain the X-ray spectrum and check if there are large amplitude and fast flux variations. Power-law emission with photon index $\sim 1.3$ and $1.6-1.7$ is often seen in redbacks in the rotation-powered state and transitional systems in the disc state, respectively, with certain X-ray luminosity values at the different states (e.g. Linares 2014; Bogdanov \& Halpern 2015; Coti Zelati et al. 2019), and the transitional systems in the disc state show their distinguishing feature of having fast switches between two different count rate levels (e.g. Patruno et al. 2014; Bogdanov \& Halpern 2015; Coti Zelati et al. 2019). Detection of such variability would certainly help establish J0935 as a candidate transitional MSP system. At last Fermi LAT observations of J0935 provide monitoring checks. If J0935 is indeed a candidate transitional system, the transition from the current subluminous disc state to a rotation-powered state would be expected. Once the $\gamma$-ray flux drops significantly in a short time period, it would likely indicate a transition, which could be verified with multiwavelength observations by searching for pulsed emission from the MSP and detecting related flux drops at X-rays and optical bands.

\section{ACKNOWLEDGEMENTS}

This paper includes data gathered with the 6.5-m Magellan telescopes located at Las Campanas Observatory, Chile. This research made use of the High Performance Computing Resource in the Core Facility for Advanced Research Computing at Shanghai Astronomical Observatory. This research was supported by the National Program on Key Research and Development Project (Grant No. 2016YFA0400804) and the National Natural Science Foundation of China (11633007, U1738131). JZ is supported by the NSFC (grants 11773067, 11403096), the Youth Innovation Promotion Association of the Chinese Academy of Sciences (grants 2018081), and the Western Light Youth Project.

\section{REFERENCES}

Abazajian K. N. et al., 2009, ApJS, 182, 543

Abdo A. A. et al., 2013, ApJS, 208, 17

Acero F. et al., 2015, ApJS, 218, 23

Ackermann M. et al., 2015, ApJ, 810, 14

Alpar M. A., Cheng A. F., Ruderman M. A., Shaham J., 1982, Nature, 300 728

Archibald A. M. et al., 2009, Science, 324, 1411

Atwood W. B. et al., 2009, ApJ, 697, 1071

Bassa C. G. et al., 2014, MNRAS, 441, 1825

Baumgartner W. H., Tueller J., Markwardt C. B., Skinner G. K., Barthelmy

S., Mushotzky R. F., Evans P. A., Gehrels N., 2013, ApJS, 207, 19

Benvenuto O. G., De Vito M. A., Horvath J. E., 2014, ApJ, 786, L7 
Bhattacharya D., van den Heuvel E. P. J., 1991, Phys. Rep., 203, 1

Bogdanov S., Halpern J. P., 2015, ApJ, 803, L27

Bogdanov S., Archibald A. M., Hessels J. W. T., Kaspi V. M., Lorimer D., McLaughlin M. A., Ransom S. M., Stairs I. H., 2011, ApJ, 742, 97

Burrows D. N. et al., 2005, Space Sci. Rev., 120, 165

Cash W., 1979, ApJ, 228, 939

Chambers K. C. et al., 2016, preprint (arXiv:1612.05560)

Chen H.-L., Chen X., Tauris T. M., Han Z., 2013, ApJ, 775, 27

Coti Zelati F. et al., 2019, A\&A, 622, A211

Coughlin M. W. et al., 2019, MNRAS, 485, 1412

Evans P. A. et al., 2009, MNRAS, 397, 1177

Flewelling H. A. et al., 2016, preprint (arXiv:1612.05243)

Fruchter A. S., Stinebring D. R., Taylor J. H., 1988, Nature, 333, 237

Gehrels N. et al., 2004, ApJ, 611, 1005

Goad M. R. et al., 2007, A\&A, 476, 1401

Halpern J. P., Strader J., Li M., 2017, ApJ, 844, 150

HI4PI Collaboration et al., 2016, A\&A, 594, A116

Kennedy M. R., Clark C. J., Voisin G., Breton R. P., 2018, MNRAS, 477, 1120

Linares M., 2014, ApJ, 795, 72

Oh K. et al., 2018, ApJS, 235, 4

Papitto A. et al., 2018, ApJ, 858, L12

Patruno A. et al., 2014, ApJ, 781, L3

Rau A. et al., 2009, PASP, 121, 1334

Roberts M. S. E., 2013, in van Leeuwen J., ed., Proc. IAU Symp. Vol. 291, Neutron Stars and Pulsars: Challenges and Opportunities after 80 years. Cambridge Univ. Press, Cambridge, p. 127

Romani R. W., Sanchez N., 2016, ApJ, 828, 7
Romani R. W., Filippenko A. V., Cenko S. B., 2015, ApJ, 804, 115

Sabbi E., Gratton R., Ferraro F. R., Bragaglia A., Possenti A., D’Amico N., Camilo F., 2003, ApJ, 589, L41

Shahbaz T. et al., 2015, MNRAS, 453, 3461

Shahbaz T., Dallilar Y., Garner A., Eikenberry S., Veledina A., Gandhi P., 2018, MNRAS, 477, 566

Stappers B. W. et al., 2014, ApJ, 790, 39

Strader J., Li K.-L., Chomiuk L., Heinke C. O., Udalski A., Peacock M., Shishkovsky L., Tremou E., 2016, ApJ, 831, 89

Strader J. et al., 2019, ApJ, 872, 42

Swihart S. J. et al., 2018, ApJ, 866, 83

Takata J. et al., 2014, ApJ, 785, 131

The Fermi-LAT collaboration, 2019, preprint (arXiv:1902.10045)

Torres D. F., Ji L., Li J., Papitto A. R., Rea N., de Oña Wilhelmi E., Zhang S., 2017, ApJ, 836, 68

van Paradijs J., McClintock J. E., 1995, in Lewin W. H. G., van Paradijs J., van den Heuvel E. P. J., eds, X-Ray Binaries. Cambridge Univ. Press, Cambridge, p. 58

Wang Z., Archibald A. M., Thorstensen J. R., Kaspi V. M., Lorimer D. R., Stairs I., Ransom S. M., 2009, ApJ, 703, 2017

Xing Y., Wang Z., 2015, ApJ, 808, 17

Xing Y., Wang Z., 2016, ApJ, 831, 143

Xing Y., Wang Z.-X., Takata J., 2018, Res. Astron. Astrophys., 18, 127

Yap Y. X., Li K. L., Kong A. K. H., Takata J., Lee J., Hui C. Y., 2019, A\&A, $621, \mathrm{~L} 9$

This paper has been typeset from a $\mathrm{T}_{\mathrm{E}} \mathrm{X} / \mathrm{L} \mathrm{A} \mathrm{E} \mathrm{X}$ file prepared by the author. 